E3S Web of Conferences 1, 41012 (2013)

DOI: $10.1051 /$ e3sconf/20130141012

C) Owned by the authors, published by EDP Sciences, 2014

\title{
Relationship between the level of zinc, lead, cadmium, nickel and chromium in hair of people with diabetes
}

\author{
$\underline{\text { F. Tadayon }}^{1 *}$, A. Abdollahi ${ }^{2}$, S. Rajabi Nia ${ }^{1}$ and R. Ostovar ${ }^{1}$ \\ ${ }^{1}$ Department of Chemistry, North Tehran Branch, Islamic Azad University, Tehran, (IRI); F_Tadayon@iau-tnb.ac.ir \\ ${ }^{2}$ Faculty of Sciences, Islamic Azad University, Khorasgan (Isfahan) Branch, 8159-158, Isfahan, Iran
}

\begin{abstract}
It has long been believed that some metals possess many adverse health effects. Recently, certain elements have been identified as essential trace elements that play an important role in the genesis and progression of several diseases. Some toxic metals have also been shown to be elevated in biological samples of diabetes mellitus patients. The status of trace elements in diabetes patients is also influenced by their diet, drugs administered and, to a large extent, by environmental factors. Pollutants due to the presence of toxic metals in environment not only enter the body by breading, water, and foodstuff accumulates in hair, but they could be adsorbed directly on the hair from environment. The aim of present study was to investigate the relationship between the level of zinc, lead, cadmium, nickel and chromium in hair samples of diabetic women from Tehran (Iran). The study population consisted of 100 women between 30 to 70 years of age from Tehran. The hair samples were washed with $1 \%(\mathrm{w} / \mathrm{v})$ (DDTC), $0.1 \mathrm{M} \mathrm{HCL}$ and deionized water. Afterwards, the hair sample dried in oven at $70^{\circ} \mathrm{C}$ for 5 hours and then digested the next day. Dry ashing digestion procedure was carried out. The concentration of elements was measured by means of an atomic absorption spectrophotometer. The statistical analysis confirmed that mean concentrations of lead and nickel did not differ significantly from the control group. The results of this study showed that the mean values of $\mathrm{Cr}$ and $\mathrm{Zn}$ were significantly decreased in scalp hair samples of diabetic patients as compared to control subjects. Hair Cd level was significantly higher in type 2 diabetic patients. Values of Pearson correlation coefficient showed positive correlation between these elements.
\end{abstract}

Keywords: Metals, Diabetes mellitus, Human hair, Atomic absorption spectrophotometry

\section{Introduction}

It has long been believed that heavy metals possess many adverse health effects. Uncontrolled industrialization has released heavy metal pollution in the world. Interest in the importance of trace elements to human health has increased considerably during last decades (1). Recently, certain elements have been identified as essential trace elements that play an important role in the genesis and progression of several diseases. Among several metals, chromium, cadmium, nickel and lead are of great importance because these elements are largely used in the metal industry including welders and alloy smelter works. There are so many factors contributing to the pathophysiology of type II diabetes, some of these factors are trace elements (2). Several studies have indicated that the deficiency and efficiency of some essential trace metals may play a role in the islet function and development of diabetes mellitus.
Some toxic metals have also been shown to be elevated in biological samples of diabetes mellitus patients (3). The status of trace elements in diabetes patients is also influenced by their diet, drugs administered and, to a large extent, by environmental factors. Many authors have reported that human hair is a good indicator of environmental pollution (4). Pollutants due to the presence of toxic metals in environment not only enter the body by breading, water, and food stuff accumulates in hair, but they could be adsorbed directly on the hair from environment (5). Trace element analysis on hair samples has been widely used to assess wildlife and human exposure to different contaminants present in the environment. Several advantages were mentioned for the use of this biological material in monitoring studies, namely: hair is a biological specimen that is easily and no invasively collected, inexpensive, and easily stored and transported to the laboratory for analysis (6). The aim of present study was to investigate the relationship between 
the level of zinc, lead, cadmium, nickel and chromium in hair samples of diabetic women from Tehran (Iran).

\section{Materials and Methods}

An atomic absorption spectrometer, Varian model spectra AA-220, was used for flame atomic absorption analysis of $\mathrm{Zn}$. For graphite furnace analysis of $\mathrm{Cd}, \mathrm{Pb}$, $\mathrm{Cr}$, and $\mathrm{Ni}$ the apparatus was equipped with a GTA-100 graphite furnace atomizer, deuterium lamp as a background corrector, and a Varian programmable sample dispenser. The study population consisted of 100 women between 35 to 70 years of age from Tehran. They answer to some questions to obtain information about age, sex, food ingestion, source of drinking water, smoking habits, alcohol consumption, health condition, medication and workplace. The hair samples were cut from the nape of the neck of each person, as close to the scalp as possible. All samples were stored individually in sealed plastic bags at room temperature. Water used was deionized from a Millipore ultra pure water system. The regents used in analysis were high quality analytical grade from Merck. Concentrated nitric acid 65\% and $30 \%$ hydrogen peroxide purchased from Merck (Darmstadt, Germany) were checked for possible trace metal contamination. Laboratory glassware were are cleaned in 5\% (V/V) nitric acid before used. Prior to analysis, hair samples were washed with $1 \%(\mathrm{w} / \mathrm{v})$ (DDTC), 0.1M HCL and deionized water. Afterwards, the hair sample dried in oven at $70^{\circ} \mathrm{C}$ for 8 hours and then digested the next day (7). Dry ashing digestion procedure was carried out. Statistical calculations were made using statistical packages SPSS (VERSION 14). The level of significance was set at $\mathrm{P}<0.05$.

\section{Results and Discussion}

In this study, Pearson correlation coefficient was obtained as a scale of correlation between two variables. Values of Pearson correlation coefficient of 5 metals in hair of diabetic people were show in Table 1.

Table 1. Pearson correlation coefficient between Elements

\begin{tabular}{cccccc}
\hline $\begin{array}{c}\text { Eleme } \\
\text { nt }\end{array}$ & $\mathbf{P b}$ & $\mathbf{C d}$ & $\mathbf{N i}$ & $\mathbf{C r}$ & $\mathbf{Z n}$ \\
\hline $\mathbf{P b}$ & 1 & 0.934 & 0.959 & 0.689 & 0.979 \\
$\mathbf{C d}$ & 0.934 & 1 & 0.939 & 0.696 & 0.941 \\
$\mathbf{N i}$ & 0.959 & 0.939 & 1 & 0.672 & 0.887 \\
\hline $\mathbf{C r}$ & 0.689 & 0.696 & 0.672 & 1 & 0.741 \\
$\mathbf{Z n}$ & 0.979 & 0.941 & 0.887 & 0.741 & 1 \\
\hline
\end{tabular}

The results show correlation between these elements was affirmative. Analysis for $\mathrm{Cr}, \mathrm{Zn}$ and $\mathrm{Cd}$ in hair revealed a significant difference between controls and patients. Hair Cd level was significantly higher in type 2 diabetic patients as compared to the control group. In

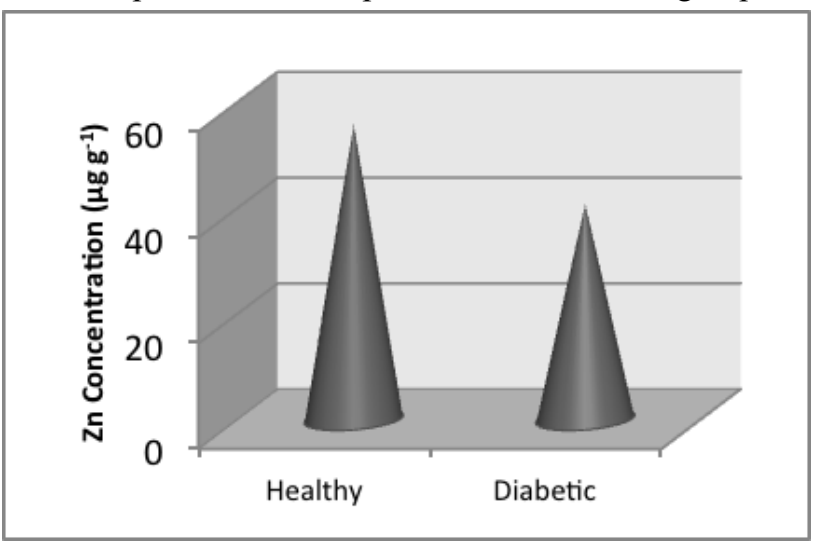

comparison with the control group, significantly

Fig. 1. Mean concentration of $\mathrm{Zn}$ in healthy and diabetic

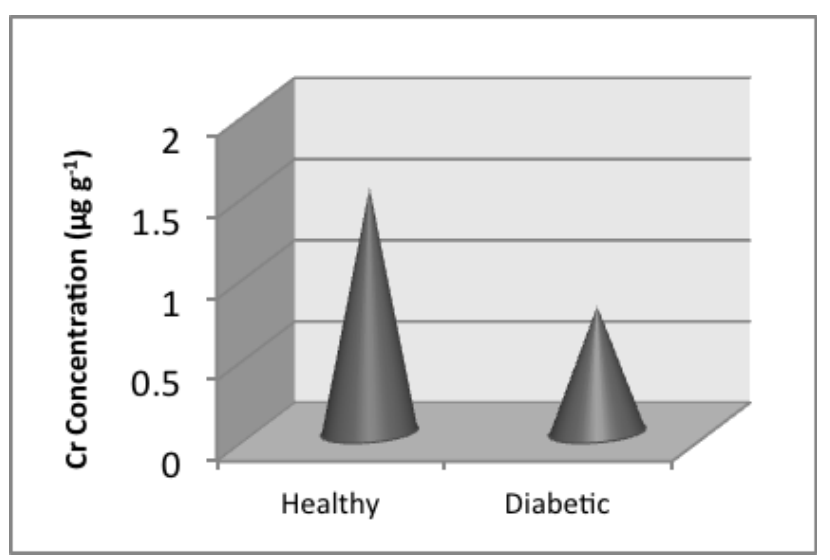

people

Fig. 2. Mean concentration of $\mathrm{Cr}$ in healthy and diabetic

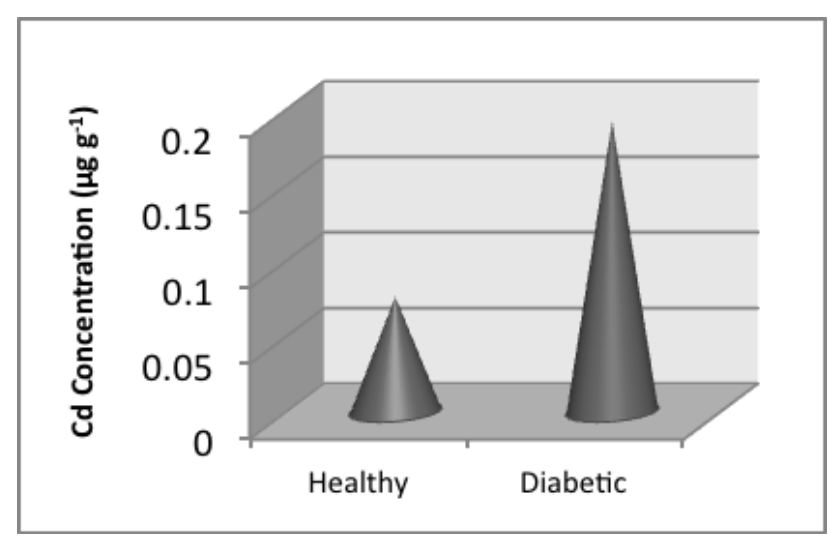

people

Fig. 3. Mean concentration of $\mathrm{Cd}$ in healthy and diabetic people

decreased $\mathrm{Cr}$ and $\mathrm{Zn}$ levels. Mean hair lead and nickel concentrations were not significantly different between the two groups. The results obtained from the concentration elements are shown in Figures 1 to 5. 


\section{Conclusion}

Metals pollutants damage organ functions and disrupt physiological homeostasis. Some toxic metals have also been shown to be elevated in biological samples of

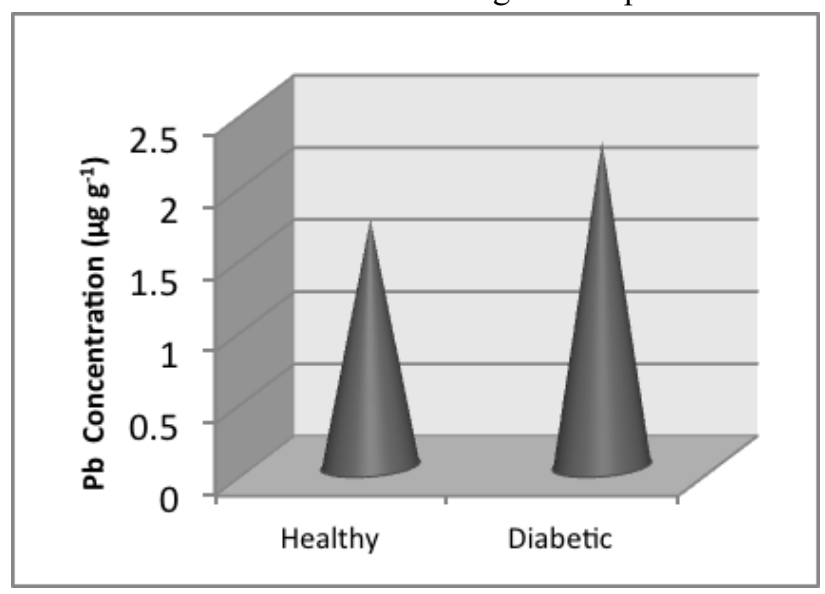

Fig. 4. Mean concentration of Ni in healthy and diabetic people

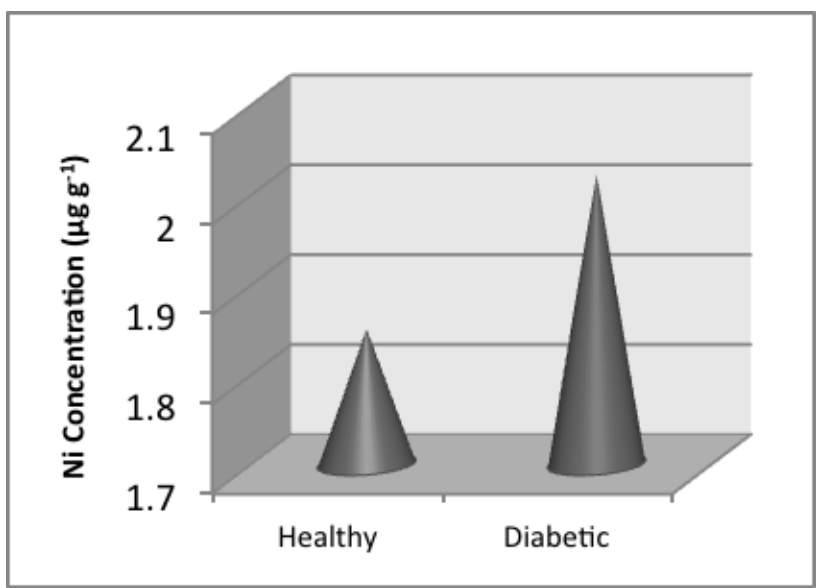

Fig. 5. Mean concentration of $\mathrm{Pb}$ in healthy and diabetic people

diabetes mellitus patients. Deficiency and efficiency of some essential trace metals may play a role in the islet function and development of diabetes mellitus. Cadmium, lead and nickel are toxic elements that have relationship with diabetes. Cadmium and lead exposure might cause diabetes. The result showed chromium and zinc are decrease in diabetic patient.

There are significant relationship between the concentrations of chromium, zinc and cadmium in diabetic and control group. The data obtained showed that there was no significant relationship between the concentrations of nickel and lead in diabetic and control group.

\section{References}

[1] Horng C.J., Tsai J.L., Horng P.H., Lin S.C., Lin S.R., Tzeng C.C., Determination of urinary lead, cadmium and nickel in steel production workers, Talanta 56 (2002) 1109.

[2] Kamal M., Salem M., Kholousi N., Ashmawy Kh., Evaluation of trace elements and Malondialdehyde levels in type II diabetes mellitus, Diabetes \& Metabolic Syndrome 3 (2009) 214-218.

[3] Wen Chen Y., Yao Yang Ch., Fa Huang Ch., Zong Hung D., Man Leung Y., Hwa Liu Sh., Heavy metals, islet function and diabetes development, Landes Bioscience 1:3(2009)169-176.

[4] Chłopicka J., Zadrodzki P., Zachwieja Z., Krooeniak M., Fłota M., Use of pattern recognition methods in the interpretation of heavy metal content lead and cadmium. in children's scalp hair, Analyst 120(1995)943-946.

[5] Wasiak W., Ciszewska W., Ciszewski A., Hair analysis. Part-1: differential pulseanodic stripping voltammetric determination of lead, cadmium, zinc and copper in human hair samples of persons in permanent contact with a polluted work place environment, Anal. Chim. Acta 335 (1996) 201207.

[6] Sreenivasa Rao K., Balaji T ., Prasada Rao T., Determination of iron, cobalt, nickel, manganese, zinc, copper, cadmium and lead in human hair by inductively coupled plasmaatomic emission spectrometry, Spectrochim. Acta 57(2002) 13331338.

[7] Tadayon F., Saber Tehrani M., Endogenous and exogenous elemental speciation in human hair by various washing procedures and determination by flam and electrothermal atomic absorption spectrometery, ASIAN J CHEM 5 (2009) 3660 3670 . 